\title{
Eficacia del propofol-fentanilo comparada con propofol- ketamina en procedimientos dolorosos mayores en un Departamento de Emergencia Pediátrica
}

\author{
Efficacy of propofol-fentanyl compared with propofol- \\ ketamine in significantly painful procedures in a Pediatric \\ Emergency Department
}

\author{
Laura Morilla', Viviana Pavlicich¹, Patricia Domínguez', Susan Portillo', Rodolfo Gauto, \\ Miki Watanabe', Mirta Mesquita ${ }^{1}$
}

\section{RESUMEN}

Objetivo: Comparar la eficacia de la combinación propofol - fentanilo (P / F) con propofol - ketamina (P / K) en procedimientos dolorosos mayores en un Departamento de Emergencias Pediátricas (DEP). Diseño: Ensayo aleatorizado, controlado, abierto, con cegamiento del evaluador. Material y Métodos: Se incluyeron pacientes de 6 meses a 18 años, con una clasificación ASA menor o igual a 3, sometidos a sedación y analgesia para la realización de procedimientos dolorosos. Intervenciones: Los sujetos fueron asignados al azar para recibir propofolfentanilo o propofol-ketamina. Se evaluaron las complicaciones. Las variables fueron: datos demográficos, tipos de procedimientos, tiempo hasta la adecuada sedación y analgesia, dosis adicionales requeridas, tiempo de recuperación y efectos adversos. Resultados: Se analizaron sesenta procedimientos (30 por grupo). No hubo diferencias en la edad, el sexo, el tipo de procedimiento, la duración de la infusión y la duración del procedimiento o el tiempo de recuperación. El tiempo para lograr la sedación (minutos) fue mayor con P / F que con P / $\mathrm{K}: 2$ (1-4 rango intercuartilo) vs. 1 (rango intercuartil a 1-1). El tiempo (minutos) para lograr una analgesia adecuada con P / F fue 2 (1-5 rango intercuartilo) vs. 1 (1-1,25 rango intercuartilo). $\mathrm{p}<0,001$. El $\mathrm{P} / \mathrm{F}$ requirió más dosis adicionales, $80 \%$ frente a $43 \%$, p < $<, 004$, y tuvo más efectos secundarios, 46, 6\% (intervalo de confianza [IC] del 95\%: $70 \%$ a $100 \%$ ) versus $20 \%$ (95\%). \% CI-2\% a 59\%) p <0,024. La proporción de efectos adversos graves con $\mathrm{P} / \mathrm{F}$ vs $\mathrm{P} / \mathrm{K}$ fue: $6 \%$ (95\% CI-1,8\% a $2 \%$ ) versus $0 \%$ (95\% CI-0 a $0 \%$ ) p 0.246. Conclusiones: Concluimos que las combinaciones de Propofol / Fentanilo y Propofol/Ketamina, son eficaces en

\section{ABSTRACT}

Objective: To compare the efficacy of the combination of propofol - fentanyl (P / F) with propofol - ketamine (P / K) in significantly painful procedures in a Pediatric Emergency Department (PED). Design: This was a randomized, controlled, open label trial, with evaluator blinding. Materials and Methods: Patients from 6 months to 18 years were included, with an ASA classification less than or equal to 3 , undergoing sedation and analgesia for significantly painful procedures. Interventions: Subjects were randomized to receive propofol-fentanyl or propofol-ketamine. Complications were evaluated. The variables were: demographic data, procedure type, time to adequate sedation and analgesia, additional doses required, recovery time and adverse effects. Results: Sixty procedures were analyzed ( 30 per group). There were no differences in age, sex, procedure type, duration of infusion, duration of the procedure or recovery time. The time to achieve sedation (minutes) was higher with $\mathrm{P} / \mathrm{F}$ than with $\mathrm{P} / \mathrm{K}$ : 2 (1-4 interquartile range) vs. 1 (interquartile range to $1-1)$. The time (minutes) to achieve adequate analgesia with $\mathrm{P} / \mathrm{F}$ was 2 (1-5 interquartile range) vs. 1 (1-1, 25 interquartile range). $\mathrm{p}<0.001$. The $\mathrm{P} / \mathrm{F}$ required more additional doses, $80 \%$ vs. $43 \%$, p $<0.004$, and had more side effects, $46.6 \%$ (95\% confidence interval [CI]: $70 \%$ to $100 \%$ ) versus $20 \%$ (95\% CI- $\%$ to $59 \%$ ), p $<0.024$. The proportion of serious adverse effects with $\mathrm{P} / \mathrm{F}$ vs P / K was: $6 \%$ (95\% CI- $1.8 \%$ to $2 \%$ ) versus $0 \%$ (95\% CI-0 to $0 \%), p=0.246$. Conclusions: We conclude that the combinations of Propofol / Fentanyl and Propofol / Ketamine are effective in achieving sedoanalgesia in significantly painful and short-duration procedures, with

\footnotetext{
${ }^{1}$ Hospital General Pediátrico "Niños de Acosta Nu". San Lorenzo, Paraguay.

Correspondencia: Laura Morilla Correo: morillaslp@gmail.com

Conflicto de intereses: Los autores declaran no poseer conflictos de interés

Recibido: 25/01/2019 Aceptado: 15/04/2019

DOI: https://doi.org/10.31698/ped.46012019003
} 
alcanzar la sedoanalgesia en procedimientos muy dolorosos y cortos. Presentando la primera combinación más efectos secundarios y requiriendo más tiempo y dosis adicionales para lograr los niveles deseados de sedoanalgesia.

Palabras claves: Sedación, analgesia, manejo del dolor, departamento emergencia pediátrica, propofol, fentanilo, ketamina.

\section{INTRODUCCIÓN}

El dolor en los niños es a menudo subestimado. Los procedimientos diagnósticos o terapéuticos causan ansiedad y miedo, lo que dificulta su realización correcta. En algunos niños, pueden producir un impacto psicológico más negativo que la propia enfermedad subyacente ${ }^{(1)}$. El dolor y la ansiedad deben ser evaluados y tratados adecuadamente. Una correcta sedación y analgesia son una prioridad para garantizar la realización técnica adecuada de los procedimientos ${ }^{(2)}$.

El procedimiento mayor o muy doloroso, es aquel al realizarlo provoca un dolor grave que puede estar o no asociado a un alto nivel de ansiedad. Nuestro objetivo será no solo la analgesia sino también la sedación, la amnesia y el control de la movilidad.

Los sedantes, analgésicos o medicamentos disociativos son necesarios en procedimientos dolorosos importantes, como cardioversión, reducción de fracturas, desbridamiento de quemaduras, toracentesis, aspiración de médula ósea, punción lumbar, sutura de heridas y acceso venoso central. Deben administrarse para inducir un estado que permita al paciente tolerar un procedimiento desagradable sin interferir con las funciones vitales. El objetivo es disminuir el nivel de conciencia, mientras se preserva el control independiente y continuo de la vía aérea ${ }^{(2)}$.

El propofol es un fármaco sedante-hipnótico con un inicio rápido y un corto tiempo de recuperación. Tiene algunos inconvenientes, como una infusión dolorosa y una disfunción cognitiva transitoria ${ }^{(3)}$. Debido a que no tiene efectos analgésicos, debe administrarse con otro medicamento durante los the P / F combination having more side effects and requiring more time and additional doses to achieve the desired levels of sedoanalgesia.

Keywords: Sedation, analgesia, pain management, pediatric emergency department, propofol, fentanyl, ketamine.

procedimientos dolorosos ${ }^{(4)}$. Los efectos adversos que requieren intervención, ocurren en aproximadamente el 2 a 5 por ciento de los niños que se someten a sedación con propofol e incluyen depresión respiratoria, apnea e hipotensión ${ }^{(4,5)}$.

La ketamina es un derivado de la fenciclidina, con propiedades sedantes, analgésicas y amnésicas. Conserva el tono muscular, protege las vías respiratorias y la respiración espontánea ${ }^{(5)}$. El tratamiento con ketamina previene la respuesta al dolor de Propofol durante la infusión y contrarresta la depresión hemodinámica ${ }^{(6,7)}$.

Cuando se usa ketamina sola, los efectos secundarios como disforia, vómitos o laringoespasmo son más comunes $^{(8)}$. La combinación de ketamina más propofol $(\mathrm{P} / \mathrm{K})$ reduce los efectos secundarios de ambos fármacos, contrarrestando los efectos hemodinámicos y respiratorios de cada uno $\mathrm{y}$ permitiendo dosis más bajas. El uso exitoso de $\mathrm{P} / \mathrm{K}$ por médicos de emergencia capacitados para realizar procedimientos dolorosos en el Departamento de Emergencia pediátrica (DEP) ha sido bien documentado $^{(9)}$.

El fentanilo es un opioide sintético que se utiliza para proporcionar analgesia en procedimientos con dolor moderado a intenso ${ }^{(10)}$. Su acción rápida (dentro de dos o tres minutos), la duración de su efecto relativamente corto (30 a 60 minutos) y su falta de liberación de histamina lo hacen preferible para procedimientos dolorosos en comparación con los opioides de acción prolongada, como la morfina ${ }^{(11,12)}$. La rigidez de la pared torácica y de la glotis son efectos adversos raros pero potencialmente fatales 
del fentanilo ${ }^{(12,13)}$. Esta rigidez muscular resulta en la incapacidad de ventilar cuando se utiliza a altas dosis $(4 \mathrm{mcg} / \mathrm{kg}$ ) o en infusión rápida. Asociarlo con propofol permite lograr los efectos deseados, utilizando dosis más pequeñas. Por otro lado, la hipoxemia, la depresión respiratoria y la apnea pueden ocurrir cuando el fentanilo se combina con sedantes como el propofol ${ }^{(13)}$.

No existen contraindicaciones absolutas para los procedimientos de sedación en niños ${ }^{(14)}$. Las contraindicaciones relativas incluyen una vía aérea difícil o comorbilidades médicas significativas (Clasificación III o superior de la American Society of Anesthesia Class) ${ }^{(14,15)}$.

El protocolo de sedación y analgesia para nuestro servicio durante procedimientos dolorosos mayores es usar ketamina o ketamina y propofol en este contexto.

El objetivo de este estudio fue determinar si la combinación de Propofol - Fentanilo es equivalente a la combinación de Ketamina - Propofol en términos de eficacia y efectos adversos.

\section{MATERIALES Y MÉTODOS}

\section{Diseño y lugar del estudio}

Estudio clínico aleatorizado, controlado, abierto con cegamiento del evaluador. Realizado en la unidad de reanimación del Departamento de Emergencias Pediátricas de un hospital universitario de atención terciaria con más de cien mil consultas por año, en los meses de junio a setiembre del 2016.

\section{Selección de participantes}

Los pacientes eran elegibles si cumplían los siguientes criterios de inclusión: edad entre 6 meses a 18 años de edad, necesidad de sedoanalgesia para uno de los siguientes procedimientos dolorosos: punción lumbar, incisión y drenaje del absceso, aspiración de médula ósea, sutura de la herida, desbridamiento de quemadura, toracocentesis, colocación del catéter venoso central, y reducción de la fractura o inmovilización de las fracturas. Tener un ASA menor o igual a 3, no tener antecedentes de reacciones adversas a la ketamina, propofol y fentanilo o alergias a la proteína del huevo y presencia del equipo de investigación entrenado en el turno en que ingresa el paciente. Este último criterio respondió a la necesidad de darle validez y fiabilidad a las mediciones. Se excluyeron los pacientes con historial de ronquidos e infecciones activas del tracto respiratorio.

Se designaron 2 médicos de emergencia con entrenamiento previo y una enfermera. Uno de los médicos se encargó de la aleatorización, la preparación y supervisión de la administración de la medicación por parte de la enfermera y el otro del llenado del instrumento de recolección de datos y la evaluación los efectos de manera ciega con respecto a la medicación administrada, utilizando las herramientas de medición. El muestro de los pacientes fue no probabilístico. Los que llenaron los criterios de inclusión fueron aleatorizados a recibir Propofol-Ketamina (grupo de referencia) o Propofol/Fentanilo (grupo estudio) La aleatorización se realizó en Microsoft Excel (versión 2015) y fueron colocados en sobres numerados secuencialmente, sellados y opacos que contenían la asignación del tratamiento.

Dosis, preparación y administración de la medicación

Se prepararon los dos fármacos de cada grupo en jeringas diferentes. En el grupo PK cada medicación se administró a una dosis de $1 \mathrm{mg} / \mathrm{kg}$. El Propofol en una concentración $10 \mathrm{mg} / \mathrm{ml}$ administrado en bolo EV en 20 a 30 segundos, la Ketamina $(50 \mathrm{mg} / \mathrm{ml})$ diluida en $5 \mathrm{ml}$ de solución fisiológica $(10 \mathrm{mg} / \mathrm{ml})$ administrado en menos de 1 minuto. En el grupo $\mathrm{PF}$, el propofol se administró a igual dosis, concentración, vía y tiempo que en el grupo PK. La dosis del fentanilo fue $1 \mu \mathrm{g} / \mathrm{kg}$, presentación 0,05 $\mathrm{mg} / \mathrm{ml}$, diluido en $10 \mathrm{ml}$ de suero fisiológico (10 $\mu \mathrm{g} / \mathrm{ml}$ ) administrado en 1 a 2 minutos.

\section{Medición de la sedación y analgesia}

El grado de sedación se midió por la escala de Ramsay, (con un rango de 1 a 6 puntos) Se consideró sedación clínica cuando el puntaje se encontraba $\leq 4$. (Ver Anexo 1)

El nivel se analgesia se midió por la escala de Campbell con un rango de 0 , ausencia de dolor a 10 dolor máximo. Se considero adecuado para el 
estudio si la escala se encontraba $\leq 3$. (Ver Anexo 2)

El tiempo de recuperación se midió desde la finalización del procedimiento utilizando la escala de Alderete (rango 0 a 10 puntos). Se consideró recuperado con un puntaje de 10 puntos. (Ver Anexo 3)

Variables medidas las siguientes variables edad, sexo, estado nutricional ( $\mathrm{P} / \mathrm{T}$ en menores de 2 años e Índice de masa corporal (IMC) en pacientes $\geq 2$ años, tipo de procedimiento, tiempo de sedación clínica (Ramsay), tiempo para alcanzar analgesia adecuada (Campbell), tiempo de recuperación, (Alderete), duración del procedimiento, necesidad de dosis adicionales. Durante todo el procedimiento los pacientes se encontraban monitorizados con medición continua de la FC, FR, saturación de Oxigeno por oximetría de pulso. La presión arterial y la escala de Glasgow se midieron en forma intermitente (al minuto, 3 y 5 min y luego hasta la recuperación total cada $5 \mathrm{~min}$ ). Figura 1.

Se evaluó la aparición de efectos adversos posibles: náuseas, vómitos, cefaleas, anafilaxia, inestabilidad hemodinámica, apnea, bradicardia, cantidad de secreciones, dolor en el lugar de la infusión, reacciones de emergencia con ketamina y laringoespamo. Se definió la hipoxia como una saturación de oxígeno inferior al $92 \%$ y la hipoxia grave cuando estaña menor a $80 \%$ El laringoespasmo se definió como un estridor inspiratorio, retracciones y movimientos de la pared torácica con inspiración. Un aumento o disminución de la presión arterial y la frecuencia cardíaca se definió como un cambio en un $25 \%$ por encima o por debajo de la medición basal, respectivamente.

Los principales punto de cortes fueron el tiempo de sedación y de analgesia alcanzado. En forma secundaria, necesidad de dosis adicionales, tiempo de recuperación, y presencia de efectos adversos.

El tamaño de la muestra se calculó utilizando variables I continuas, comparando dos medias, con un riesgo de error $\alpha$ de 0.05 y una potencia del $80 \%$. Para detectar diferencias entre ambos grupos y considerando un porcentaje de abandono del $5 \%$, se debe reclutar 30 pacientes en cada grupo. Los datos fueron cargados y analizados en SPSSv21.Las variables cualitativas se expresaron en porcentajes y las cuantitativas en medianas o medias con DE de acuerdo a su distribución. La asociación entre las variables se realizo mediante la prueba de chi cuadrado para las cualitativas y la T Student o Mann Whitney de acuerdo a su distribución. Se consideró un error alfa inferior al $5 \%$.

El protocolo fue aprobado por el comité de ética de la institución con consentimiento informado de los padres o encargados.

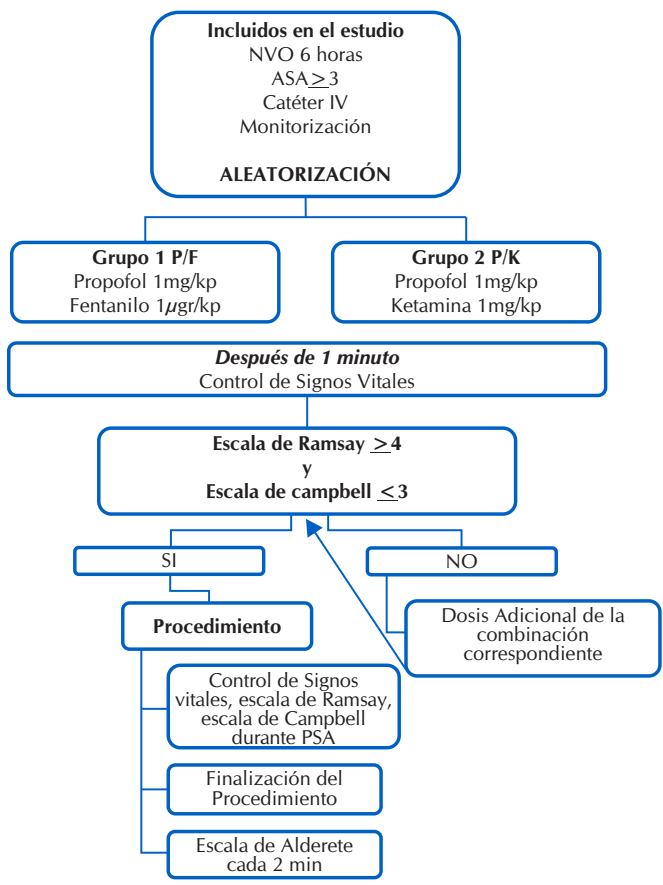

Figura 1. Diagrama de flujo NVO (nada por vía oral), ASA (Clasificación de la Sociedad Americana de Anestesia), PSA (procedimientos de sedoanalgesia).

\section{RESULTADOS}

En el periodo de estudio 60 pacientes llenaron los criterios de inclusión. Treinta en cada grupo como se observa en el flujograma. Figura 2.

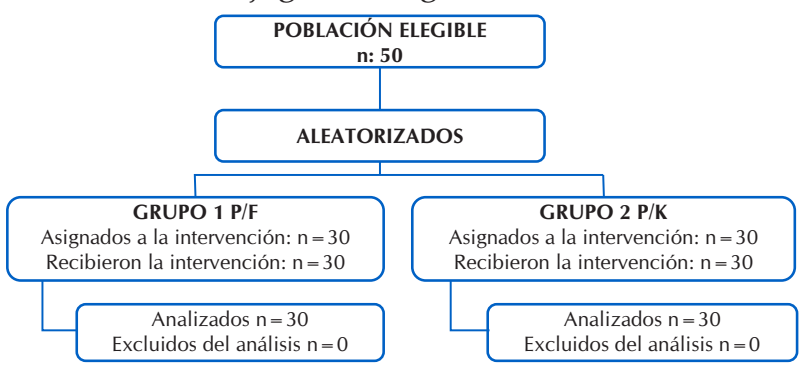

Figura 2. Diagrama de Flujo. 
No hubo diferencias entre los grupos en cuanto a edad, sexo, estado nutricional, tiempo de infusión y duración y tipo de procedimientos realizados. Tabla 1.

Durante la infusión y el procedimiento no hubo diferencias significativas entre los grupos de estudio en la variación de los signos vitales (frecuencia cardíaca, frecuencia respiratoria, saturación de oxígeno) A los 3 minutos de iniciada la infusión, la media de la presión arterial sistólica determinada en el grupo P / F fue de $112 \mathrm{~mm} \mathrm{Hg}$ y en el grupo P/K $126 \mathrm{~mm} \mathrm{Hg}$ (p: 0.02). Ningún paciente presento hipotensión. Un paciente en el $\mathrm{P} / \mathrm{F}$ presentó una disminución superior al 25\% y otro paciente en el grupo $\mathrm{P} / \mathrm{K}$ tuvo un aumento superior al $25 \%$ de sus mediciones de presión arterial respecto a la determinación basal. Figura 3.

El tiempo para lograr una sedación y analgesia adecuada, el tiempo de recuperación del paciente y el número de dosis adicionales están expresados en la Tabla 2.

El grupo $\mathrm{P} / \mathrm{F}$ tuvo más efectos secundarios, $43.3 \%$ vs. $16.6 \%(\mathrm{p}<0.024)$. El tiempo medio de recuperación $( \pm$ DE) entre los grupos no mostró diferencias (Tabla 2). Los efectos secundarios están descriptos en la Tabla 3. El tiempo medio para el desarrollo de efectos secundarios fue de 3,31 $\pm 2,4$ minutos en el grupo P / F y 3,40 $\pm 3,9$ minutos en el grupo $\mathrm{P} / \mathrm{K}$. Un paciente en el grupo de $\mathrm{P} / \mathrm{K}$ tuvo vómitos a los 60 minutos.

Las intervenciones de rescate realizadas en el $\mathrm{P} / \mathrm{F}$ fueron: en un paciente, ventilación con presión positiva, en tres pacientes reposicionamiento de las vías respiratorias y oxigenación, y en siete pacientes solo alineación de las vías áreas. En el $\mathrm{P} / \mathrm{K}$, dos pacientes requirieron alineación de las vías áreas.

No se requirió escalonamiento en los cuidados, intubación, ventilación asistida, reanimación inotrópica y con líquidos en ningún paciente. Ningún paciente necesitó hospitalización debido a la sedación. No se requirieron antídotos.

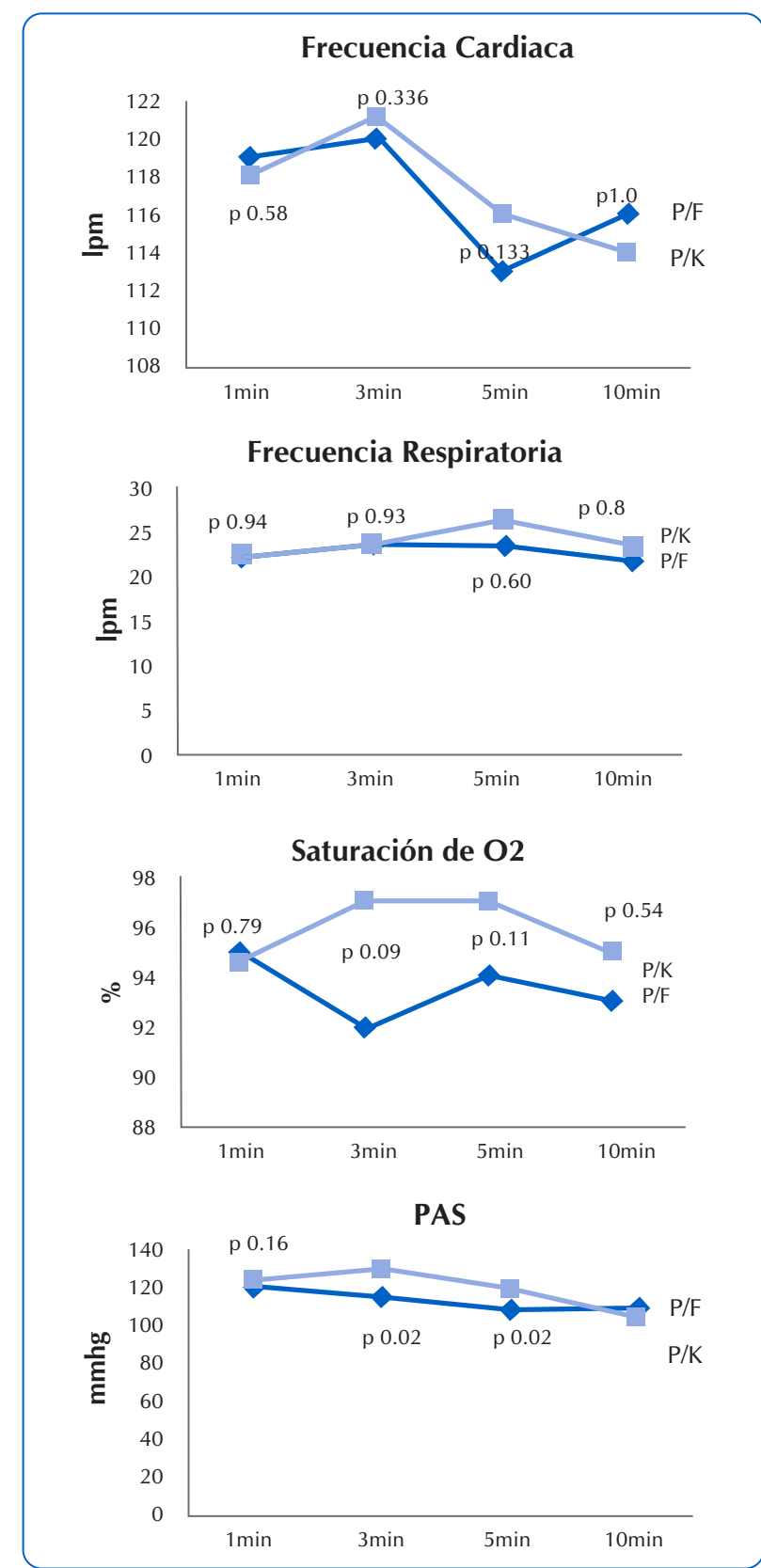

Figura 3. En la abscisa el tiempo del procedimiento en minutos, en la ordenada: A) Frecuencia cardiaca: latidos por minuto B) Frecuencia respiratoria: respiraciones por minuto C) Saturación de oxigeno D) Presión arterial sistólica en $\mathrm{mm} \mathrm{Hg}$. 
Tabla 1. Comparación de las características entre los grupos P/Fy P/K.

\begin{tabular}{|c|c|c|c|}
\hline Variables & $P / F(n=30)$ & $P / K(n=)$ & $p$ \\
\hline Edad (media \pm DS ) & $5.1 \pm 4.3$ & $5,7 \pm 4,5$ &, 79 \\
\hline \multicolumn{4}{|l|}{ Sexo $n(\%)$} \\
\hline Femenino & $12(40)$ & $17(57)$ & \multirow[t]{2}{*}{, 15} \\
\hline Masculino & $18(60)$ & $13(43)$ & \\
\hline $\begin{array}{l}\text { * IMC } \\
\text { (mediana, rango) }\end{array}$ & $16,2(14,7-18,7)$ & $16(14,7-18,4)$ &, 882 \\
\hline \multicolumn{4}{|l|}{ Procedimientos $\mathrm{n}(\%)$} \\
\hline Punción lumbar & $8(27)$ & $9(30)$ & \multirow{6}{*}{, 73} \\
\hline$* *$ PAMO & $9(30)$ & $5(17)$ & \\
\hline Sutura & $8(27)$ & $7(23)$ & \\
\hline Drenaje de abscesos & $3(10)$ & $5(17)$ & \\
\hline Quemaduras & $1(3)$ & $1(3)$ & \\
\hline Otros & $1(3)$ & $3(10)$ & \\
\hline $\begin{array}{l}\text { Duración de la infusión, } \\
\text { minutos (media } \pm \text { DS ) }\end{array}$ & $1,53 \pm 0,6$ & $1,50 \pm 0,57$ & 100 \\
\hline $\begin{array}{l}\text { Duración de los minutos del } \\
\text { procedimiento (media } \pm \text { DS })\end{array}$ & $3,92 \pm 3,1$ & $2,97 \pm 1,7$ & 473 \\
\hline
\end{tabular}

Tabla 2. Comparación del tiempo para alcanzar la sedación (Ramsay $\geq 4$ ) y la analgesia (Campbell $\leq 3)$ y la recuperación (Aldrete 10), número de dosis adicionales requeridas por cada grupo, frecuencia de los efectos adversos.

\begin{tabular}{|c|c|c|c|}
\hline & $P / F(n=30)$ & $P / K(n=)$ & $p$ \\
\hline $\begin{array}{l}\text { Puntuación Ramsay }>4 \text {, } \\
\text { minutos, mediana (IQR) }\end{array}$ & $2(1-4)$ & $1(1-1)$ & $0,001^{*}$ \\
\hline $\begin{array}{l}\text { Puntuación Campbell } \leq 3 \\
\text { minutos, mediana (rango) }\end{array}$ & $2(1-5)$ & $1(1-1,25)$ & $0,001^{*}$ \\
\hline $\begin{array}{l}\text { Tiempo de recuperación, } \\
\text { minutos, mediana (rango) }\end{array}$ & $4(4-10)$ & $6(4-10)$ & $0,394^{*}$ \\
\hline $\begin{array}{l}\text { Dosis adicionales } \\
\text { n (\%) (IC } 95 \%)\end{array}$ & $24(80)(70-100)$ & $13(43)(2-59)$ &, $004^{* *}$ \\
\hline $\begin{array}{l}\text { Número de dosis adicionales } \\
\text { (media } \pm \text { DS) (IC del 95\%) }\end{array}$ & $1,83 \pm 0,9(0,5-1,2)$ & $1 \pm 0,5(0,29-0,71)$ & $0,041^{*}$ \\
\hline $\begin{array}{l}\text { Efectos adversos } \\
\text { n (\%) (IC } 95 \%)\end{array}$ & $14(46,6)(30-63)$ & $6(20)(9-37)$ & $0,024^{* *}$ \\
\hline $\begin{array}{l}\text { Efectos adversos graves } \\
\text { n (\%) (IC 95\%) }\end{array}$ & $2(6)(1,8-2)$ & $0(0-0)$ & $0,246 * *$ \\
\hline
\end{tabular}

Tabla 3. Comparación de efectos adversos entre P / F y P/K.

\begin{tabular}{lcc}
\hline Tipo & $\mathbf{P} / \mathbf{F}(\mathbf{n}=\mathbf{3 0})$ & $\mathbf{P} / \mathbf{K}(\mathbf{n}=\mathbf{3 0})$ \\
\hline Hipoxemia & $\mathrm{n}(\%)$ & \\
Hipoxemia severa & $9(30)$ & $2(6)$ \\
Dolor en el sitio de infusión & $2(6)$ & $0(0)$ \\
Vómito & $2(6)$ & $0(0)$ \\
Laringoespasmo & $0(0)$ & $1(3)$ \\
Urticaria & $0(0)$ & $1(3)$ \\
BP aumenta 25\% desde el inicio & $0(0)$ & $1(3)$ \\
Reducción de la PA en un 25\% con & $0(0)$ & $1(3)$ \\
respecto al inicio. & $1(3)$ & $0(3)$ \\
Bradicardia &
\end{tabular}

\section{DISCUSIÓN}

En este estudio se encontró que la combinación de PK en procedimientos dolorosos mayores en el DEP logró alcanzar niveles de sedoanalgesia más rápido, con menos dosis adicionales y menos efectos secundarios. Sin embargo, ambas combinaciones lograron niveles efectivos de sedoanalgesia y tuvieron tiempos de recuperación similares. Este resultado podría explicarse porque la ketamina, pero no el fentanilo, agregó efectos sedantes al propofol. No hubo diferencias en la frecuencia o características de los tipos de procedimientos realizados, ni en la duración del procedimiento o en el tiempo de infusión de la medicación que influya en los resultados. Andolfatto G y cols. en dos estudios en el DEP utilizando propofol/ketamina, demostró que es una mezcla eficaz, con un tiempo de recuperación corto, pocos eventos adversos y altos índices de satisfacción del personal sanitario y de los pacientes ${ }^{(8,16)}$.

El fentanilo se ha combinado con el propofol en circunstancias similares para agregar propiedades analgésicas en procedimientos realizados fuera del quirófano. Investigamos la posibilidad de usar propofol/fentanilo de la misma manera y alternativamente al propofol/ketamina. Fue necesario administrar más dosis para alcanzar el efecto deseado y comprobamos una mayor frecuencia de efectos colaterales con esta combinación.

La duración media de los procedimientos realizados en este estudio fue inferior a cinco minutos. Creemos que esta breve duración se atribuye al tipo de procedimientos que usualmente se realizan en el DEP y a que fueron realizados por médicos con experiencia e iniciados en pacientes con nivel adecuado de sedoanalgesia.

Se analizó el tiempo requerido para alcanzar los niveles de analgesia y sedación adecuados para comenzar los procedimientos para así evaluar la eficacia comparativa de las combinaciones. El grupo de pacientes tratados con ketamina/propofol alcanzó estos niveles más rápidamente, con similitud al estudio publicado por Thota et al. ${ }^{(17)}$. Creemos que aunque la diferencia en el tiempo de alcanzar la sedoanalgesia entre los grupos es estadísticamente significativa, la misma no tendría una significancia clínica en los pacientes estables presentados en este estudio, ni impacto en la gestión del DEP. Esta diferencia si podría ser relevante en podría ser relevante en procedimientos de 
emergencia como el drenaje de un neumotórax a tensión.

La hipoxemia fue el efecto adverso más frecuente en el grupo P/F. Un paciente de este grupo presentó hipoxemia grave con bradicardia y requirió ventilación con presión positiva con bolsa y mascarilla durante menos de un minuto antes de la recuperación completa.

Encontramos un aumento de la presión arterial en el grupo PK y una disminución de la saturación en el grupo PF. Pensamos que esta última cobraría significancia al aumentar el número de pacientes estudiados y que ambos efectos son los propios de la droga que se asoció al propofol en cada grupo.

El niño que tuvo laringoespasmo era un niño obeso. Los niños obesos deben considerarse con mayor riesgo de sedación fuera del entorno de la sala de operaciones $^{(18,19)}$. Los vómitos en los pacientes que recibieron ketamina no fueron muy frecuentes aunque el ondansetrón no se utilizó de forma rutinaria para prevenirlos. Tampoco notamos salivación excesiva ni reacciones de emergencia en pacientes a los que se les administró ketamina. La presión arterial mayor después del inicio de la

\section{REFERENCIAS}

1. Walls R, Murphy MF. Manual of Emergency Airway Management. 3 ed. Philadelphia, PA: Lippincott Williams \&Wilkins; 2004.

2. Hug CC Jr, McLeskey CH, Nahrwold ML, Roizen MF, Stanley TH, Thisted RA, et al. Hemodynamic effects of propofol: data from over 25,000 patients. Anesth. Analg. 1993;77(4 Suppl):S21-S29.

3. Mortero RF, Clark LD, Tolan MM, Metz RJ, Tsueda K, Sheppard RA. The effects of small-dose ketamine on propofol sedation: respiration, postoperative mood, perception, cognition, and pain. Anesth. Analg. 2001; 92(6):1465-1469.

4. Suzuki S, Masamune T, Nonaka A, Masamune T, Kumazawa T. Pre-treatment with ketamine reduces incidence and severity of pain on propofol injection. infusión en el grupo 2 podría deberse al efecto hipertensivo de la ketamina.

\section{Limitaciones}

Este estudio presenta algunas limitaciones. Los procedimientos fueron de corta duración y no urgentes, los resultados podrían variar con tiempos de procedimiento más largos y en situaciones de emergencia.

El estudio no proporciona ninguna información sobre la experiencia del paciente durante el procedimiento o la satisfacción de la familia ${ }^{(20)}$.

\section{CONCLUSIONES}

Concluimos que las combinaciones de Propofol / Fentanilo y Propofol/Ketamina, a las dosis que se administraron y en pacientes con estabilidad cardiorrespiratoria, son eficaces en alcanzar la sedoanalgesia en procedimientos muy dolorosos y cortos.

Sin embargo Propofol /Fentanilo presento más efectos secundarios y requirió más tiempo y más dosis adicionales para lograr los niveles deseados de sedoanalgesia.

Masui. 2002;51(8):140-143.

5. Bassett KE, Anderson JL, Pribble CG, Guenther E. Propofol for procedural sedation in children in the emergency department. Ann. Emerg. Med. 2003; 42(6):773-782.

6. Green SM, Krauss B. The semantics of ketamine. Ann. Emerg. Med. 2000;36(5):480-482.

7. Bowdle TA, Radant AD, Cowley DS, Kharasch ED, Strassman RJ, Roy-Byrne PP. Psychedelic effects of ketamine in healthy volunteers: relationship to steady-state plasma concentrations. Anesthesiology. 1998; 88(1):82-88.

8. Willman EV, Andolfatto G. A prospective evaluation of "ketofol" (ketamine/propofol combination) for procedural sedation and analgesia in the emergency 
department. Ann. Emerg. Med. 2007;49(1):23-30.

9. Frizelle HP, Duranteau J, Samii K. A comparison of propofol with a propofol-ketamine combination for sedation during spinal anesthesia. Anesth. Analg. 1997; 84(6):1318-1322.

10. Zempsky WT, Cravero JP, American Academy of Pediatrics Committee on Pediatric Emergency Medicine and Section on Anesthesiology and Pain Medicine. Relief of pain and anxiety in pediatric patients in emergency medical systems. Pediatrics. 2004; 114(5):1348-1356.

11. Finn M, Harris D. Intranasal fentanyl for analgesia in the paediatric Emergency Department. Emerg. Med. J. 2010;27(4):300-301.

12. Gomar C, Fernandez C. Epidural analgesiaanaesthesia in obstetrics. Eur. J. Anaesthesiol. 2000;17(9):542-558.

13. Hegenbarth MA, American Academy of Pediatrics Committee on Drugs. Preparing for pediatric emergencies: drugs to consider. Pediatrics. 2008; 121(2):433-443.

14. Grunwell JR, McCracken C, Fortenberry J, Stockwell J, Kamat P. Risk factors leading to failed procedural sedation in children outside the operating room. Pediatr. Emerg. Care. 2014;30(6):381-387.

15. Shavit I, Leder M, Cohen DM. Sedation provider practice variation: a survey analysis of pediatric emergency subspecialists and fellows. Pediatr. Emerg. Care. 2010;26(10):742-747.

16. Andolfatto G, Willman E. A prospective case series of single-syringe ketamine-propofol (Ketofol) for emergency department procedural sedation and analgesia in adults. Acad. Emerg. Med.2011;18(3):237-245.

17. Thota RS, Ambardekar M, Likhate P. Conscious sedation for middle ear surgeries: A comparison between fentanyl-propofol and fentanyl-midazolam infusion. Saudi J. Anaesth. 2015; 9(2):117-121.

18. Grunwell JR, Travers C, McCracken CE, Scherrer PD, Stormorken AG, Chumpitazi CE, et al. Procedural sedation outside of the operating room using ketamine in 22,645 Children: A Report From the Pediatric Sedation Research Consortium. Pediatr Crit Care Med. Diciembre 2016; 17(12): 1109-1116.

19. Bhatt M, Johnson DW, Chan J, Taljaard M, Barrowman $\mathrm{N}$, Farion $\mathrm{KJ}$, et al. Risk factors for adverse events in Emergency Department Procedural Sedation for Children. JAMA Pediatr. 2017; 171(10):957-964.

20. Williams MR, Nayshtut M, Hoefnagel A, McKeown A, Carlson DW, Cravero J. et al. Efficacy Outcome Measures for Pediatric Procedural Sedation Clinical Trials: An ACTTION Systematic Review. Anesth Analg. 2018;126(3):956-967. 


\section{ANEXOS}

Anexo 1. Escala de Ramsay: sedación

Nivel 0: ausencia de sedación; Nivel 1-3: sedación consciente; Nivel 4-6: sedación profunda

\begin{tabular}{|l|}
\hline \multicolumn{1}{|c|}{ ESCALA RAMSAY } \\
\hline Nivel 0: Agitado, ansioso, intranquilo. \\
Nivel 1: tranquilo, cooperador y despierto. Orientado. \\
Nivel 2: Dormido. Abre los ojos con el ruido ambiental. \\
Nivel 3: Dormido. Abre los ojos abre si se le llama. \\
Nivel 4: Dormido. Abre los ojos con estímulos físicos. \\
Nivel 5: Dormido. No abre los ojos pero se mueve y reacciona con estímulos físicos. \\
Nivel 6: inconsciente y sin respuesta.
\end{tabular}

Anexo 2. Escala de Campbell: dolor en paciente sedado: Sin dolor 0; Dolor leve - moderado 1-3; Dolor moderado-grave 4- 6; Dolor muy intenso $>6$. Objetivo $\leq 3$

\begin{tabular}{|c|c|c|c|c|}
\hline & 0 & 1 & 2 & $\begin{array}{c}\text { Puntuación } \\
\text { parcial }\end{array}$ \\
\hline Musculatura facial & Relajada & $\begin{array}{l}\text { En tensión, ceño fruncido y/o } \\
\text { mueca de dolor }\end{array}$ & $\begin{array}{l}\text { Ceño fruncido de forma } \\
\text { habitual y/o dientes apretados }\end{array}$ & \\
\hline "Tranquilidad" & $\begin{array}{l}\text { Tranquilo, relajado, } \\
\text { movimientos normales }\end{array}$ & $\begin{array}{l}\text { Movimientos ocasionales de } \\
\text { inquietud y/o posición }\end{array}$ & $\begin{array}{l}\text { Movimientos frecuentes, } \\
\text { incluyendo cabeza o } \\
\text { extremidades }\end{array}$ & \\
\hline Tono muscular* & Normal & $\begin{array}{l}\text { Aumentado. Flexión de } \\
\text { dedos de manos y/o pies }\end{array}$ & Rígido & \\
\hline Respuesta verbal** & Normal & $\begin{array}{l}\text { Quejas, Iloros, quejidos o } \\
\text { gruñidos ocasionales }\end{array}$ & $\begin{array}{l}\text { Quejas, lloros, quejidos o } \\
\text { gruñidos frecuentes }\end{array}$ & \\
\hline Confortabilidad & $\begin{array}{l}\text { Confortable y/o } \\
\text { tranquilo }\end{array}$ & $\begin{array}{l}\text { Se tranquiliza con el tacto y/o } \\
\text { la voz. Fácil de distraer }\end{array}$ & $\begin{array}{l}\text { Difícil de confortar con el } \\
\text { tacto o hablándole }\end{array}$ & \\
\hline \multicolumn{4}{|c|}{$\begin{array}{l}\text { Puntuación Escala de Campbell } \\
\text { *En caso de lesión medular o hemiplejía valorar el lado sano } \\
\text { **Puede ser poco valorable en vía aérea artificial }\end{array}$} & $/ 10$ \\
\hline
\end{tabular}

Anexo 3. Escala de recuperación: Aldrete

\begin{tabular}{|c|c|c|}
\hline Categorías & Ítems & Puntos \\
\hline Actividad Motora & $\begin{array}{l}\text { Posibilidad par amover } 4 \text { extremidades de forma espontánea o en respuesta a ordenes } \\
\text { Posibilidad para mover } 2 \text { extremidades de forma espontánea o en respuesta a ordenes } \\
\text { Imposibilidad para mover } 4 \text { extremidades de forma espontánea o en respuesta a ordenes }\end{array}$ & $\begin{array}{l}2 \\
\mathbf{1} \\
\mathbf{0}\end{array}$ \\
\hline Respiración & $\begin{array}{l}\text { Posibilidad para respirar profundamente y toser frecuentemente } \\
\text { Disnea o respiración limitada } \\
\text { Sin respuesta }\end{array}$ & $\begin{array}{l}2 \\
1 \\
0\end{array}$ \\
\hline Circulación & $\begin{array}{l}\text { Presión arterial }=20 \% \text { del nivel pre anestésico } \\
\text { Presión arterial entre } 20 \% \text { y } 49 \% \text { del valor anestésico } \\
\text { Presión arterial }=50 \% \text { del valor pre anestésico }\end{array}$ & $\begin{array}{l}\mathbf{2} \\
\mathbf{1} \\
\mathbf{0}\end{array}$ \\
\hline Consistencia & $\begin{array}{l}\text { Plenamente despierto } \\
\text { Responde cuando se llama } \\
\text { Sin respuesta }\end{array}$ & $\begin{array}{l}2 \\
1 \\
0\end{array}$ \\
\hline Color & $\begin{array}{l}\text { Rosado } \\
\text { Pálido } \\
\text { Cianótico }\end{array}$ & $\begin{array}{l}2 \\
1 \\
0\end{array}$ \\
\hline
\end{tabular}

